\section{Planejamento da qualidade nas unidades de saúde da família, utilizando o Desdobramento da Função Qualidade (QFD)}

\author{
Quality planning of Family Health Units using \\ Quality Function Deployment (QFD)
}

\author{
${ }^{1}$ Faculdade de Odontologia \\ de Piracicaba, Universidade \\ Estadual de Campinas, \\ Piracicaba, Brasil. \\ Correspondência \\ L. F. Volpato \\ Faculdade de Odontologia \\ de Piracicaba, Universidade \\ Estadual de Campinas. \\ Rua dos Engenhos de \\ Piracicaba 30, Piracicaba, SP \\ 13405-163, Brasil. \\ lucianavolpato@superig.com.br
}

\begin{abstract}
Quality is an indispensible requirement in the health field, and its pursuit is necessary in order to meet demands by a population that is aware of its rights, as part of the essence of good work relations, and to decrease technological costs. Quality thus involves all parties to the process (users and professionals), and is no longer merely an attribute of the health service. This study aimed to verify the possibility of quality planning in the Family Health Units, using Quality Function Deployment (QFD). QFD plans quality according to user satisfaction, involving staff professionals and identifying new approaches to improve work processes. Development of the array, called the House of Quality, is this method's most important characteristics. The results show a similarity between the quality demanded by users and the quality planned by professionals. The current study showed that QFD is an efficient tool for quality planning in public health services.
\end{abstract}

Quality of Health Care; Health Services; Health Personnel; Quality Management; Health Planning
Luciana Fernandes Volpato ${ }^{1}$

Marcelo de Castro Meneghim 1

Antonio Carlos Pereira 1

Gláucia Maria Bovi Ambrosano 1

\section{Introdução}

A Organização Mundial da Saúde (OMS) conceitua saúde como um estado de completo bemestar físico, mental e social, e não apenas a ausência de doença 1 ; desta forma, pode-se pensar que não há uma pessoa absolutamente saudável e que não necessite de serviços de saúde. A Constituição Federal de 1988, criadora do Sistema Único de Saúde (SUS), introduz o conceito de saúde ampliado, afirmando que " a saúde é direito de todos e dever do Estado, garantido mediante políticas sociais e econômicas que visem à redução do risco de doença e de outros agravos e ao acesso universal e igualitário às ações e serviços para a promoção, proteção e recuperação" (Artigo 196, p. 91).

Na sua estruturação, o SUS possui os princípios doutrinários (universalidade, equidade e integralidade) e organizativos (descentralização, regionalização e hierarquização, resolutividade e controle social), sendo regulamentado pela Lei Orgânica da Saúde (Lei $n^{\circ}$. 8.080/90), que dispõe sobre a capacidade de resolução dos serviços em todos os níveis de assistência, partindo do fundamento de que a assistência à saúde é livre à iniciativa privada. A mesma Lei, no Artigo 22, diz ainda que "na prestação de serviços privados de assistência à saúde, serão observados os princípios éticos e as normas expedidas pelo órgão de direção do SUS quanto às condições de seu funcionamento" 2. Portanto, é necessário aprimorar 
e desenvolver a qualidade em qualquer setor de assistência à saúde, seja público ou privado.

A criação do SUS visava a romper com o modelo até então vigente de atenção à saúde, de caráter curativo, discriminatório e mercantilista; contudo, após vinte anos, o quadro da saúde no Brasil não é muito diferente daquele que o movimento buscava superar. De acordo com Roncalli 3 , não é de hoje que esse sistema vem sendo atacado, devido às mutilações ocorridas nas suas características fundamentais: universalidade, integralidade e garantia da saúde como direito do cidadão.

A população, por meio constitucional, teve garantido o direito de acesso à saúde, entretanto o sistema público de saúde é precário, e a busca pela qualidade se faz necessária diante das profundas desigualdades sociais, péssimas condições de vida e de saúde da população, má alocação dos recursos, ineficiência, custos crescentes e desigualdade nas condições de acesso dos usuários 4 . Não alheia aos acontecimentos, a imprensa exibe constantemente a falência do sistema público de saúde; a ineficácia e ineficiência deste podem ser observadas nas imensas filas e nas macas espalhadas pelos corredores 3 dos hospitais.

A qualidade que está sendo discutida referese ao cuidado integral, como o acolhimento, o vínculo, a equipe de trabalho, pois se trata de um tema essencial em qualquer instituição que busque excelência no atendimento ao usuário. Nos serviços de saúde, essa busca ocorre principalmente no setor privado 5 , de forma que a participação do usuário unido aos profissionais traduza um serviço mais humano e eficaz.

O setor público é o maior prestador de bens e serviços à população, possuindo responsabilidades que dependem da qualidade, da agilidade e da localização desses serviços, no entanto ainda focaliza sua própria existência, deixando a qualidade em segundo plano ${ }^{6}$. Segundo Paiva \& Gomes 7, quando se pretende administrar um serviço de saúde tendo como perspectiva a qualidade, deve-se focalizar o indivíduo e não a doença, provando que os indivíduos estão mais interessados na prevenção e promoção da saúde.

Assim, a atenção básica é considerada uma das colunas da organização do sistema de saúde, sendo o primeiro contato do usuário. As unidades de saúde da família estão inseridas no nível básico de atenção e apresentam potencial para resolver a maioria dos problemas, uma vez que não se restringem ao diagnóstico de uma doença e ao seu tratamento, mas realizam ações de prevenção e promoção da saúde ${ }^{8,9}$. Diante desses fatores, o planejamento da qualidade na atenção primária se faz necessário e deve ser fundamen- tado nos princípios doutrinários e organizativos do SUS, entretanto esses serviços não possuem mecanismos sistematizados e validados para seu monitoramento e avaliação 10 .

Para auxiliar no planejamento da qualidade, existem várias alternativas de ferramentas. Entre elas, destaca-se o Desdobramento da Função Qualidade (QFD), metodologia que, embora pouco utilizada na área da saúde, pode ser definida como um processo estruturado que procura planejar a qualidade baseando-se não somente na satisfação do usuário, contando com o envolvimento dos profissionais 11 e da instituição 12,13 .

A ferramenta QFD foi desenvolvida no Japão por Yoji Akao e Shiguero Mizuno, entre 1960 e 1965, período de grande crescimento da indústria japonesa 14 . Na ocasião, a indústria automobilística nipônica fazia constante alteração nos modelos e lançava novos veículos, necessitando de um método que garantisse a qualidade do produto desde a fase inicial do projeto, e a solução foi o QFD 15.

Em virtude da elevada concorrência mundial, na década de 80, o QFD passou a ser utilizado em vários países europeus e nos Estados Unidos; chegou ao Brasil na década de 90. Após vinte anos de divulgação no solo brasileiro, seu uso é relativamente recente, havendo carência de dados sobre sua aplicação 16. Atualmente, no exterior, o QFD está presente em segmentos voltados à prestação de serviços de saúde 17; no Brasil, há poucos estudos que o utilizam para o planejamento da qualidade nessa área.

Segundo Barley 18, a estrutura de uma organização influencia o desempenho dos profissionais e vice-versa. De acordo com esse enfoque, é necessário resgatar o desempenho dos profissionais nos serviços de saúde para que tenham disponível uma estrutura de trabalho adequada para atender suas necessidades e de seus usuários 5,13 .

Os outros métodos, em sua grande maioria, limitam-se à aplicação de manuais, metas e planos de melhoria sem a visão global do ambiente; avaliam a qualidade restringindo-se a indicadores quantitativos, como taxa de mortalidade, atendimento à demanda e, raras vezes, satisfação do usuário 19. O QFD, por sua vez, planeja a qualidade conforme a satisfação do usuário, envolvendo os profissionais e identificando caminhos para aperfeiçoar os processos de trabalho. Utiliza ferramentas e matrizes, de modo que a construção da Casa da Qualidade é um dos aspectos mais importante ${ }^{12}$, pois correlaciona os requisitos aspirados pelo usuário com as características do serviço necessárias para satisfazer os profissionais, possibilitando estabelecer de metas, sanar as barreiras inerentes à organiza- 
ção e auxiliar no processo de planejamento dos serviços $11,14,20$.

Diante do exposto, o objetivo desta pesquisa foi verificar a possibilidade de planejar a qualidade dos serviços de saúde nas unidades de saúde da família por meio da metodologia QFD. Em face da necessidade de aprimoramento nos processos de análise da qualidade na área da saúde, no Brasil, levantou-se a hipótese de que essa ferramenta seja um instrumento capaz de oportunizar contribuições na referida área.

\section{Metodologia}

Este estudo é do tipo exploratório-descritivo e utilizou-se a técnica de amostragem por conglomerados.

Seguindo um dos princípios adotados pelo SUS - regionalização das 32 unidades de saúde da família existentes na cidade de Piracicaba, São Paulo, Brasil -, foram selecionadas para a pesquisa quatro unidades localizadas em bairros com índices de exclusão social de -1 a $-0,5$ e de $-0,5$ a 0 .

Esse índice funciona como uma espécie de controle social do bem-estar e da qualidade de vida da população, estando algumas variáveis ligadas a ele: violência, desigualdade, nível de escolaridade e emprego formal 21 . Os resultados dos índices podem variar de - 1 a 1 e foram agrupados em classes para separar as melhores condições de inclusão (mais próximos de 1) dos casos mais graves de exclusão social (mais próximos de -1).

A amostra do estudo constituiu-se de 240 voluntários, entre profissionais e usuários das quatro unidades, denominadas 1, 2, 3 e 4. Desta amostra, 40 são profissionais de saúde vinculados às unidades e 200 são usuários, compostos por 50 voluntários selecionados em cada uma das unidades.

Os critérios de inclusão para os profissionais foram os seguintes: todos que atuavam nas unidades quando a entrevista foi realizada, ou seja, médico, enfermeiro, técnicos de enfermagem, agentes comunitários de saúde, cirurgião-dentista e atendente de consultório dentário; ambos os gêneros e faixa etária de 18-70 anos. Para os usuários, os critérios adotados foram os seguintes: indivíduos vinculados à unidade; ambos os gêneros; faixa etária de 18-70 anos. Neste caso, os menores de 18 foram excluídos por não serem emancipados, enquanto os pacientes idosos deveriam ser independentes ou parcialmente dependentes, orientados no tempo e espaço, além de ter capacidade de verbalização.

A coleta de dados teve início após a obtenção de parecer favorável da Secretaria da Saúde do Município de Piracicaba e dos responsáveis pelas equipes das unidades de saúde da família. O período da coleta compreendeu os meses de março a junho de 2009, e a pesquisa foi realizada em dias aleatórios para obter uma amostra heterogênea.

No momento da coleta dos dados, o usuário foi comunicado sobre a pesquisa, seu propósito, garantia do anonimato e convidado a participar; caso a resposta fosse positiva, assinava o termo de consentimento livre e esclarecido para a pesquisa em duas vias. A seguir, foi realizada a coleta das informações nas próprias unidades, em local reservado, evitando inibição, constrangimentos e interferências nas respostas.

Os dados, em cada uma das unidades, foram coletados em duas fases: na primeira, por meio de entrevistas com os profissionais; na segunda, mediante a aplicação de questionários aos usuários. Cada uma das unidades teve seu próprio questionário e, após a coleta dos dados, iniciouse a construção da Casa da Qualidade.

O estudo foi aprovado pelo Comitê de Ética em Pesquisa da Faculdade de Odontologia de Piracicaba, Universidade Estadual de Campinas, sob processo no. 129/2008.

\section{Construção da Casa da Qualidade}

A primeira etapa da construção da Casa da Qualidade foi definir a amostra dos profissionais e usuários como descrito na metodologia. Volpato et al. 22 mencionam que, além da definição da amostra, há outras etapas necessárias para construir a Casa da Qualidade.

\section{Identificação da qualidade exigida pelo usuário}

Para identificar a qualidade exigida a fim de satisfazer as necessidades dos usuários foi realizada uma entrevista, em cada uma das unidades, com a participação de todos os profissionais das equipes, cujo propósito foi responder à questão: "Quais os itens de qualidade necessários para satisfazer os usuários desta unidade de saúde da família?".

Conforme a resposta dos profissionais, a qualidade exigida teve como base as reclamações mais frequentes dos usuários em relação ao serviço. Posteriormente, esta qualidade foi confrontada com as necessidades exigidas pelos usuários, com o objetivo de verificar se haveria incongruências. O resultado desse confronto revelou não haver divergências na identificação da qualidade exigida, efetivamente, pelos usuários. 
As respostas obtidas durante a identificação da qualidade exigida foram desdobradas em níveis (Tabela 1) e descritas na Casa da Qualidade (Figura 1).

\section{Ranqueamento da qualidade exigida}

Com base nos dados do desdobramento da qualidade exigida, foram elaborados questionários para serem aplicados aos usuários. Nos questionários, existiam duas colunas, uma relacionada à importância que o usuário atribui ao serviço, onde havia a pergunta "Qual o grau de importância que você atribui ao serviço?", e outra mencionando a avaliação sobre o serviço ofertado, onde era questionado "Como você avalia o serviço desta unidade de saúde da família?”.

Durante a aplicação dos questionários, os usuários foram orientados a responder às questões da seguinte maneira: ranquear cada uma das perguntas relacionadas com o grau de importância que o serviço possui. Desta forma, os usuários atribuíram valores de 1 a 5, de acordo com seu grau de satisfação com os serviços ofertados pelas unidades, sendo: 1 - nenhuma importância; 2 - pouca importância; 3 - alguma importância; 4 - importante; 5 - muito importante. Na compilação dos dados, foi realizada a média aritmética, e o valor resultante foi descrito no campo Grau de Importância da Casa da Qualidade (Figura 1), determinando as qualidades exigidas mais importantes.

O mesmo procedimento foi realizado para a segunda questão: a cada um dos itens de qualidade do serviço avaliado pelo usuário, atribuiuse um valor de 1 a 5, sendo: 1 - péssimo; 2 - ruim; 3 - regular; 4 - bom; 5 - ótimo. Os valores resultantes obtidos pela média aritmética foram utilizados no campo da Qualidade Planejada, descrita posteriormente.

\section{Estabelecimento das características} da qualidade

O profissional responsável pelo serviço (enfermeiro) utilizou as qualidades exigidas pelos usuários para definir as características de qualidade do serviço, ou seja, as características importantes para os profissionais e que podem auxiliar na melhoria dos serviços de saúde. Cada uma das qualidades exigidas foi correlacionada a pelo menos uma característica da qualidade (Tabela 2).
Após determinar as características da qualidade, o profissional correlacionou, individualmente, as qualidades exigidas com as características de qualidade, respondendo à pergunta: "A característica atende as necessidades dos usuários?”. No interior de cada célula, para determinar a correlação, foram utilizados os símbolos: forte $\odot$, médio $\mathbf{O}$, fraco $\triangle$ e inexistente (em branco); cada um desses símbolos apresenta um valor: forte $\odot$ -9; médio $O$ - 3; fraco $\triangle-1$; inexistente-zero.

Em seguida, os dados foram convertidos multiplicando-se o valor do símbolo pelo valor do Grau de Importância (fornecido pelo usuário), sendo o resultado registrado no interior de cada célula. Esse procedimento foi realizado em todas as células que apresentaram algum símbolo.

\section{Qualidade projetada}

A qualidade projetada indica a ordem de importância das características da qualidade (profissional), baseada nas necessidades dos usuários, e fornece alvos técnicos aos profissionais.

Para estabelecer a qualidade projetada, calcularam-se os pesos absoluto e relativo. $\mathrm{O}$ peso absoluto foi calculado somando-se os valores das células referentes a cada uma das colunas das características de qualidade. $\mathrm{O}$ peso relativo foi calculado com base nos pesos absolutos, resultando em percentuais para cada característica, determinando as características mais importantes para a melhoria dos serviços.

\section{Qualidade planejada}

Esta etapa indica a importância das qualidades exigidas e estratégias de satisfação do usuário em relação ao serviço. Portanto, por meio da avaliação dos usuários, a qualidade planejada permite estabelecer melhorias em cada item da qualidade exigida.

O profissional responsável pela unidade estabeleceu o argumento técnico, ou seja, a importância de cada item de qualidade no serviço, estabelecendo pesos de 1,0 (neutro), 1,2 (importante) e 1,5 (muito importante). Para estabelecer o plano, o profissional atribuiu valores de 1 a 5 aos itens de qualidade exigida de acordo com seu grau de importância no serviço. O valor máximo é o ideal.

A partir do plano de melhoria, determinouse: índice de melhoria (divide-se o plano pela avaliação do usuário), peso absoluto (o grau de importância é multiplicado pelo índice de melhoria e pelo argumento técnico) e peso relativo (percentual de cada item avaliado). 
Exemplo de desdobramento da qualidade exigida.

\begin{tabular}{lll}
\hline Primário & Secundário & Terciário \\
\hline \multirow{2}{*}{ Acesso } & Agendar consultas & Facilidade para agendar consultas \\
& Encaminhar aos serviços especializados & Facilidade no encaminhamento \\
& & Facilidade para agendar consultas \\
& & nos serviços especializados \\
Recepção & Atendimento & Ágil \\
& Atenção & Cordial/Simpático/Atencioso \\
\hline
\end{tabular}

\section{Correlação das características da qualidade}

Para finalizar a construção da Casa da Qualidade, o profissional responsável pela unidade correlacionou, entre si, as características da qualidade no telhado da matriz, indicando o quanto uma característica interfere na outra. Observou-se a correlação entre duas características, como mostra a Figura 1, através dos pesos: fortemente positivo (++); positivo (+); negativo (-); ou fortemente negativo (--).

\section{Resultado e discussão}

O processo de planejamento da qualidade na área da saúde envolve quem produz e quem utiliza os serviços. O planejamento da qualidade não é definitivo e estático, visto que o desenvolvimento do conhecimento e a dinâmica social exigem melhorias contínuas na maneira de oferecer o serviço 24 .

Para cada uma das unidades de saúde da família foi confeccionada uma Casa da Qualidade, pois os serviços apresentam características próprias e seus resultados diferem entre si.

Os resultados obtidos através da Casa da Qualidade serão apresentados e discutidos por etapas.

\section{Identificação da qualidade exigida}

A qualidade exigida fornecida pelos profissionais foi adicionada na Casa da Qualidade no item correspondente.

Alguns itens de qualidade exigida foram comuns a todos os serviços: facilidade para agendar consultas no serviço e nos tratamentos especializados (oftalmologista, ortopedista, entre outros) e postura de atendimento (atencioso, cordial, ágil). Há outros itens mencionados em mais de um serviço, tais como: facilidade para encami- nhamento de tratamento especializado; pontualidade no atendimento; confiança e valorização da equipe; recursos disponíveis para a promoção da saúde e manutenção do serviço (limpeza, pequenos reparos e consertos). Outras unidades apresentaram características próprias: melhores adequações da estrutura física e elevado número de famílias cadastradas.

O estudo realizado por Tengan 25 nas Unidades Básicas de Saúde e Centro de Especialidade Odontológica, utilizando a mesma ferramenta de avaliação da qualidade, difere do presente estudo, que é focalizado nas unidades de saúde da família. Entretanto, obteve itens de qualidade exigida semelhantes: facilidade de agendamento no serviço, facilidade para encaminhamento de tratamento especializado, postura de atendimento, pontualidade e confiança na equipe.

\section{$\underline{\text { Ranqueamento da qualidade exigida }}$}

Os usuários ranquearam o grau de importância dos itens descritos na qualidade exigida, ou seja, determinaram a importância que cada um dos itens tem nos serviços de saúde.

Para identificar as qualidades exigidas mais importantes aos usuários e seus respectivos graus de importância, as informações foram descritas por unidade:

- Unidade 1: facilidade para agendar consulta no serviço e no tratamento especializado, facilidade no encaminhamento de tratamento especializado, atendimento ágil, pontualidade no atendimento e valorização da equipe; todos os itens tiveram o grau de importância no valor de 4,70 ;

- Unidade 2: facilidade para agendar consulta de tratamento especializado e confiança na equipe (grau de importância correspondente a 4,70 nos dois itens);

- Unidade 3: facilidade para agendar consulta no serviço, pontualidade no atendimento, reso- 
Figura 1

Casa da Qualidade.

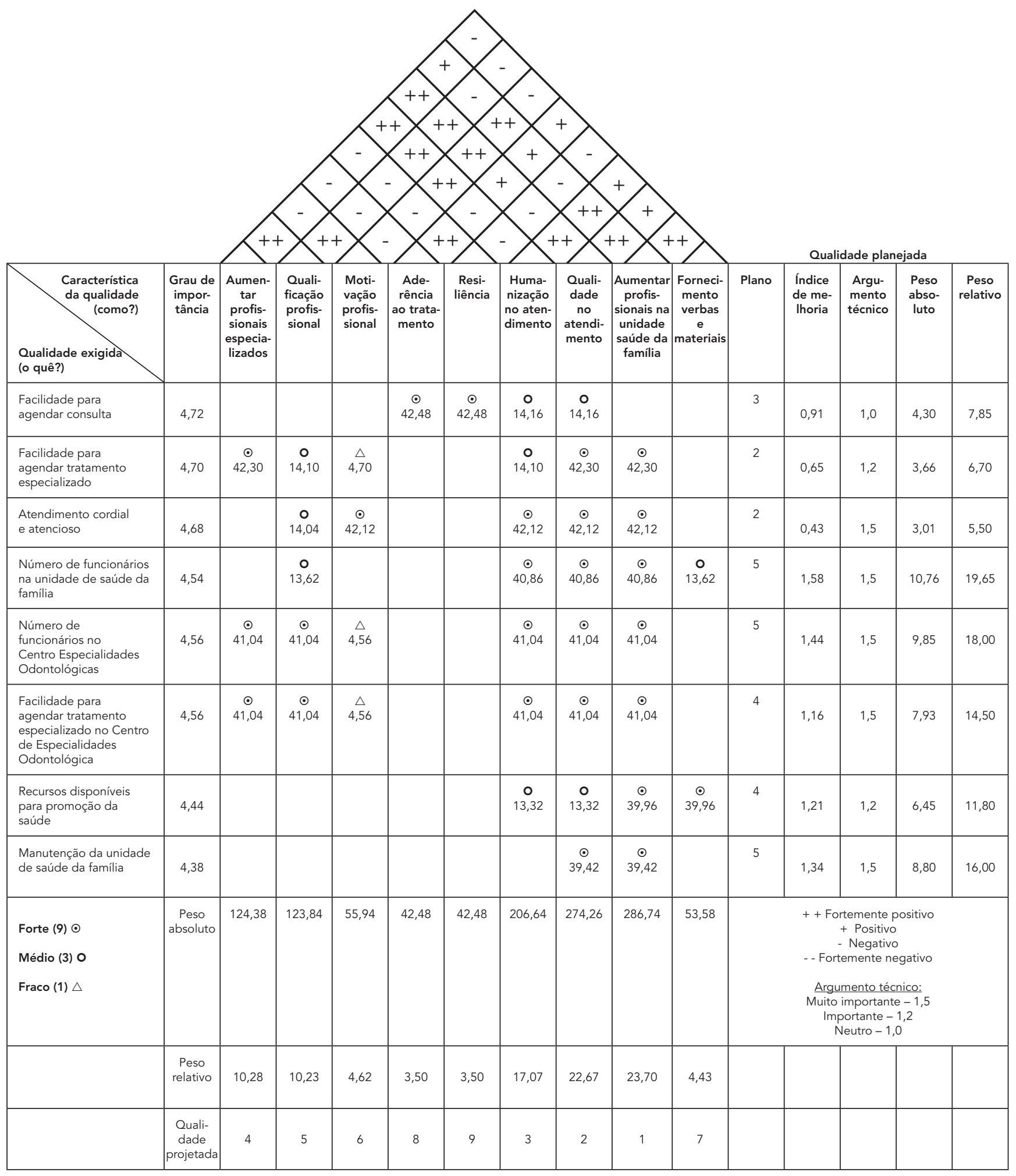

Fonte: adaptado de Milan et al. 23 . 
Exemplo de estabelecimento das características da qualidade.

\begin{tabular}{ll}
\hline Qualidade exigida & Características da qualidade \\
\hline Facilidade para agendar consultas & $\begin{array}{l}\text { Número de vagas oferecidas } \\
\text { Número de profissionais }\end{array}$ \\
\hline
\end{tabular}

lução dos problemas e confiança na equipe (grau de importância de 4,70);

- unidade 4: facilidade para agendar consulta no serviço (com o valor de 4,72 para o grau de importância).

Os estudos de Kloetzel et al. 25, Matsuda et al. 4 e Mendes et al. 26 corroboram com os resultados desta pesquisa e mencionam a importância da pontualidade no atendimento. Os achados também estão de acordo com as pesquisas de Schwartz et al. 27 e Tengan 24 , que identificaram a facilidade para agendar consulta de tratamento especializado e no serviço como aspectos de grande importância ao usuário. Franco \& Campos 28 mostram que a demora no atendimento, excesso de pessoas para serem atendidas e dificuldade para conseguir consulta em serviço especializado são os maiores problemas enfrentados pelos usuários.

\section{Estabelecimento das características da qualidade}

As características da qualidade, definidas pelos profissionais, foram:

- Unidade 1: aumento no número das vagas oferecidas e de profissionais, motivação profissional, incentivo profissional e financeiro, educação continuada e ampliação na estrutura da unidade;

- Unidade 2: diminuição no número de pessoas cadastradas, aumento no número de profissionais especializados, consulta de acompanhantes, características de personalidade, motivação profissional, acolhimento, educação continuada, fornecimento de verbas e materiais e construção de outra unidade;

- Unidade 3: aumento no número de vagas oferecidas e de profissionais, rapidez no acesso, pontualidade, aumento no número de equipamentos de informática, incentivo profissional e financeiro, educação continuada, diminuição da rotatividade profissional e usuários satisfeitos;

- Unidade 4: aumento no número de profissionais especializados, qualificação profissional, motivação profissional, aderência ao tratamento, resiliência (habilidade humana de se adaptar diante de tragédias, traumas, adversidades, privações e de fatores estressantes significativos e corriqueiros da vida), humanização e qualidade no atendimento (capacidade do serviço para satisfazer as expectativas do usuário, podendo envolver: presteza, competência, credibilidade, confiabilidade, disponibilidade dos profissionais, organização e iniciativa), aumento no número de profissionais e fornecimento de verbas e materiais.

Algumas características da qualidade foram iguais para todas as unidades, como aumento no número de profissionais, educação continuada ou qualificação profissional e motivação profissional.

\section{Características da qualidade mais significativas}

Para verificar as características da qualidade mais importantes, é necessário correlacionar, separadamente, todos os itens da característica da qualidade com o item da qualidade exigida.

Na Casa da Qualidade da unidade 1, por exemplo, a correlação entre aumentar o número de vagas oferecidas e a facilidade em agendar consultas é forte $(\odot)$, uma vez que, se houver mais vagas disponíveis, mais consultas serão agendadas. A correlação entre aumentar número de profissionais e pontualidade no tratamento é média (0), pois, com o aumento do número de profissionais, provavelmente os usuários não terão que aguardar muito tempo para serem consultados. A relação existente entre aumentar número de profissionais e facilidade no encaminhamento para tratamento especializado é fraca $(\triangle)$, já que o número de profissionais não interfere de forma significativa no encaminhamento para o tratamento especializado. E, para finalizar, não há relação entre o incentivo profissional e financeiro e a estrutura da unidade, assim esta célula não foi preenchida. 


\section{Qualidade projetada}

As qualidades projetadas, ou seja, os requisitos necessários para a melhoria do serviço do ponto de vista profissional, foram descritas de acordo com a classificação nas unidades de saúde da família, conforme Tabela 3.

Ao analisar os pontos mencionados pelos profissionais das unidades em estudo, identificaram-se quatro níveis de qualidade projetada, expressa em ordem decrescente de importância: - Com $100 \%$, estão os itens de qualidade mencionados em todas as unidades: educação continuada, incluindo o item qualificação profissional; necessidade de aumentar o número de profissionais, aumento que se subdivide em duas categorias: profissionais (75\%) e profissionais especializados (50\%); são profissionais os agentes comunitários, médicos generalistas, enfermeiros, ao passo que profissionais especializados são ortopedistas, oftalmologistas, entre outros.

- Com 75\%, os itens de qualidade mencionados em três unidades: motivação profissional.

- Com 50\%, os mencionados em duas unidades: incentivo profissional e financeiro, fornecimento de verbas e materiais, aumento no número de vagas oferecidas.
- Pontos menos significativos (elementos particularizados em uma unidade): ampliação da estrutura da unidade, diminuição do número de pessoas cadastradas, acolhimento, características de personalidade, construção de outra unidade, consulta de acompanhante, usuários satisfeitos, pontualidade na consulta, diminuição da rotatividade profissional, rapidez no acesso, aumento do número de equipamentos de informática, qualidade no atendimento, humanização no atendimento, aderência ao tratamento e resiliência.

\section{Qualidade planejada}

Por meio da avaliação dos usuários, a qualidade planejada permite estabelecer melhorias em cada item da qualidade exigida. A qualidade planejada referente aos quatro serviços pesquisados pode ser observada na Tabela 4 .

Ao analisar as referidas unidades com suas respectivas qualidades planejadas pelos usuários, pode-se chegar à identificação de quatro níveis de qualidade planejada, expressa em níveis de importância:

- Com $100 \%$ estão os seguintes itens: facilidade para agendar consultas, facilidade para agendar

Tabela 3

Classificação das qualidades projetadas nas unidades de saúde da família.

\begin{tabular}{|c|c|}
\hline Unidade 1 & Unidade 2 \\
\hline 1. Educação continuada & 1. Educação continuada \\
\hline 2. Motivação profissional & 2. Diminuição no número de pessoas cadastradas \\
\hline 3. Incentivo profissional e financeiro & 3. Acolhimento \\
\hline 4. Aumento no número de profissionais & 4. Características de personalidade \\
\hline 5. Aumento no número de vagas oferecidas & 5. Motivação profissional \\
\hline \multirow[t]{4}{*}{ 6. Ampliação na estrutura da unidade } & 6. Aumento no número de profissionais especializados \\
\hline & 7. Construção de outra unidade \\
\hline & 8. Consulta de acompanhante \\
\hline & 9. Fornecimento de verbas e materiais \\
\hline Unidade 3 & Unidade 4 \\
\hline 1. Usuários satisfeitos & 1. Aumento no número de profissionais \\
\hline 2. Aumento no número de profissionais & 2- Qualidade no atendimento \\
\hline 3. Aumento no número de vagas oferecidas & 3. Humanização no atendimento \\
\hline 4. Pontualidade na consulta & 4. Aumento no número de profissionais especializados \\
\hline 5. Diminuição da rotatividade profissional & 5. Qualificação profissional \\
\hline 6. Rapidez no acesso & 6. Motivação profissional \\
\hline 7. Educação continuada & 7. Fornecimento de verbas e materiais \\
\hline 8. Incentivo profissional e financeiro & 8. Aderência ao tratamento \\
\hline 9. Aumento no número de equipamentos de informática & 9. Resiliência \\
\hline
\end{tabular}


Classificação da qualidade planejada nas unidades de saúde da família.

\begin{tabular}{|c|c|c|c|}
\hline Unidade 1 & $\%$ & Unidade 2 & $\%$ \\
\hline 1. Manutenção da unidade & 14,90 & 1. Número de famílias cadastradas & 16,30 \\
\hline 2. Confiança na equipe & 14,32 & 2. Facilidade para agendar consulta de tratamento especializado & 13,44 \\
\hline 3. Facilidade no encaminhamento para tratamento especializado & 12,30 & 3. Pontualidade no atendimento & 11,28 \\
\hline 4. Facilidade para agendar consulta de tratamento especializado & 12,20 & 4. Facilidade para agendar consultas & 11,17 \\
\hline 5. Facilidade para agendar consulta & 10,20 & 6. Recursos disponíveis para promoção da saúde & 10,18 \\
\hline 6. Atendimento cordial e atencioso & 8,25 & 5. Resolução dos problemas & 9,98 \\
\hline 7. Valorização da equipe & 8,10 & 7. Confiança na equipe & 9,30 \\
\hline 8. Estrutura física da unidade & 7,51 & 8. Valorização da equipe & 9,02 \\
\hline 9. Pontualidade no atendimento & 6,80 & 9. Atendimento cordial e atencioso & 8,63 \\
\hline 10. Atendimento ágil & 5,42 & & \\
\hline Unidade 3 & $\%$ & Unidade 4 & $\%$ \\
\hline 1. Resolução dos problemas & 15,33 & 1. Número de funcionários na unidade & 19,65 \\
\hline 2. Confiança na equipe & 14,50 & $\begin{array}{l}\text { 2. Número de funcionários no Centro de Especialidades } \\
\text { Odontológicas }\end{array}$ & 18,00 \\
\hline 3. Facilidade no encaminhamento para tratamento especializado & 13,85 & 3. Manutenção da unidade & 16,00 \\
\hline 4. Facilidade para agendar consulta de tratamento especializado & 13,64 & $\begin{array}{l}\text { 4. Facilidade para agendar tratamento especializado no } \\
\text { Centro de Especialidades Odontológicas }\end{array}$ & 14,50 \\
\hline 5. Facilidade para agendar consulta & 11,52 & 5. Recursos disponíveis para promoção da saúde & 11,80 \\
\hline 6. Atendimento cordial e atencioso & 8,60 & 6. Facilidade para agendar consulta & 7,85 \\
\hline 7. Pontualidade no atendimento & 8,55 & 7. Facilidade para agendar tratamento especializado & 6,70 \\
\hline 8. Valorização da equipe & 8,44 & 8. Atendimento cordial e atencioso & 5,50 \\
\hline 9. Atendimento ágil & 5,57 & & \\
\hline
\end{tabular}

consultas em tratamento especializado, atendimento cordial e atencioso.

- Com 75\%: confiança e valorização da equipe, pontualidade no atendimento.

- Com 50\%: manutenção da unidade de saúde, facilidade no encaminhamento para tratamento especializado, recursos disponíveis para promoção da saúde, atendimento ágil, resolução dos problemas.

- Pontos menos significativos (elementos particularizados em algumas unidades): estrutura física da unidade, número de famílias cadastradas, número de funcionários na unidade, número de funcionários no Centro de Especialidades Odontológicas (CEO).

\section{Correlação das características da qualidade}

A correlação das características da qualidade está demonstrada no telhado da Casa da Qualidade e mostra a correlação entre os indicadores de qualidade.

As unidades 1, 3 e 4 apresentam prevalência da correlação fortemente positiva (++), demonstrando que, se houver ocorrência em um dos indicadores, seja positiva, seja negativa, o outro indicador também será afetado. Exemplo: aumentar vagas oferecidas e aumentar número de profissionais.

Entretanto, na unidade 2, ocorreu maior prevalência da correlação fortemente negativa (--), mostrando que podem ocorrer alterações nos indicadores, mas a maioria não será afetada.

\section{Relação entre os princípios do SUS e a qualidade planejada e projetada}

Os resultados referentes à qualidade planejada (usuários) e projetada (profissionais), quando relacionados com os princípios doutrinários e organizativos do SUS, possibilitam a percepção de que tais princípios não alcançam uma aplicabilidade satisfatória, uma vez que usuários e profissionais apresentam queixas sobre a gestão das unidades de saúde da família.

O descompasso do princípio da universalidade com a qualidade planejada evidencia-se no interesse dos usuários por maior facilidade no agendamento de consultas na unidade onde estão cadastrados. 
Aplicando-se essa mesma compreensão ao princípio da equidade e da integralidade, podese constatar a insatisfação dos usuários no encaminhamento para tratamento especializado e na demora para agendar esse tipo de consulta.

O princípio da descentralização também apresenta descompasso com a qualidade projetada, já que os profissionais estão insatisfeitos com a falta de manutenção da unidade, com o fornecimento de verbas e materiais para a promoção, proteção e recuperação da saúde, verificando-se, ainda, necessidade de ampliação da estrutura física.

O princípio da regionalização e hierarquização contrasta com a qualidade planejada e projetada: usuários e profissionais apontam o elevado número de famílias cadastradas e a necessidade de uma nova unidade de saúde da família.

Por fim, o princípio da resolutividade também não está sendo efetivo, pois os usuários reclamaram da ausência de pontualidade, agilidade no atendimento e necessidade de resolução dos problemas.

\section{Considerações finais}

A presente pesquisa verificou que a ferramenta QFD pode ser utilizada no planejamento da qualidade nos serviços públicos de saúde, especificamente nas unidades de saúde da família.

Foi possível constatar a relação existente entre as qualidades projetadas (obtidas através das características da qualidade fornecidas pelos profissionais) e planejadas (obtidas através das qualidades exigidas fornecidas pelos usuários). Dessa forma, nota-se que profissionais e usuários têm as mesmas necessidades de mudanças, uma vez que os dois itens de qualidade analisados por diferentes atores dentro das unidades de saúde apresentaram classificação semelhante.

A qualidade projetada e a planejada foram classificadas e expressas em quatro níveis de importância, havendo semelhanças entre as características.

No nível mais importante (100\%), os itens da qualidade projetada que requerem alterações no serviço são: educação continuada e necessidade de aumento no número de profissionais; por sua vez, os itens da qualidade planejada, ou seja, as necessidades mais importantes dos usuários, as quais demandam alterações na estrutura são: facilidade para agendar consultas na unidade e no tratamento especializado, atendimento cordial e atencioso. Nota-se um forte grau de similaridade entre a qualidade projetada (profissionais) e a qualidade planejada (usuários).
Em um segundo nível de importância, foi possível constatar que $75 \%$ dos profissionais destacaram como qualidade projetada a motivação profissional. Na qualidade planejada, os usuários destacaram, também com $75 \%$, confiança, valorização da equipe e pontualidade no atendimento, isto é, o que os usuários entenderam como aspecto importante apresenta íntima relação com a motivação profissional projetada pelos profissionais. Repete-se a similaridade.

Posteriormente, no que se refere à qualidade projetada pelos profissionais, constatou-se que $50 \%$ destacaram os seguintes itens: incentivo profissional e financeiro, fornecimento de verbas e materiais, aumento do número de vagas oferecidas. Paralelamente, na qualidade planejada pelos usuários, verificou-se que 50\% destacaram: manutenção da unidade, facilidade no encaminhamento para tratamento especializado, recursos disponíveis para promoção da saúde, atendimento ágil e resolução dos problemas. Mais uma vez, destaca-se a similitude entre ambas.

Em um quarto momento, foi possível verificar que houve elementos presentes tanto na qualidade projetada pelos profissionais, quanto na qualidade planejada pelos usuários, e são questões muito particulares de cada unidade. Essa característica é importante para se verificar que há necessidades e expectativas generalizantes $\mathrm{e}$ particulares.

Ao pensar no tema da qualidade dos serviços, um caminho de atuação seria atingir em primeiro lugar os aspectos que apresentam $100 \%$, tanto para profissionais, quanto para usuários; em seguida, contemplar sucessivamente outros aspectos, até chegar ao nível das necessidades locais. Esse mesmo raciocínio pode ser empregado de maneira inversa, partindo do particular para o geral. Mas esta é uma decisão a ser tomada pelos gestores do sistema.

No presente estudo, é visível a similaridade entre a qualidade exigida pelos usuários e a qualidade planejada pelos profissionais. Essa informação é relevante na medida em que aponta aspectos que devem merecer atenção dos gestores dos serviços de saúde.

Os estudos de Matsuda et al. 5 e Tengan 24 indicam que o QFD demonstrou ser um método eficaz na realização do planejamento da qualidade nos serviços de saúde, como hospitais, enfermagem e odontologia. O presente estudo nas unidades de saúde da família confirma a hipótese inicial de que tal ferramenta é um instrumento capaz de oportunizar contribuições na referida área, além de auxiliar nas estratégias que devem ser adotadas para a melhoria da instituição. 


\section{Resumo}

A qualidade é um requisito indispensável na área da saúde, e sua procura se faz presente em virtude da necessidade de atender as exigências de uma população ciente de seus direitos, da essência das boas relações no trabalho e diminuição dos custos tecnológicos. Assim, a qualidade envolve todos que fazem parte do processo (usuários e profissionais), deixando de ser um atributo do serviço. Esta pesquisa tem como objetivo verificar a possibilidade de planejar a qualidade nas unidades de saúde da família, por meio do Desdobramento da Função Qualidade (QFD). Este planeja a qualidade segundo a satisfação do usuário, envolvendo os profissionais da instituição e identificando novos caminhos para aperfeiçoar os processos de trabalho. A construção da matriz, denominada Casa da Qualidade, é o aspecto mais importante desse método. Os resultados mostram que há similaridade entre a qualidade exigida pelos usuários e a qualidade planejada pelos profissionais. $O$ presente estudo comprovou que a ferramenta QFD constitui-se em instrumento eficiente no planejamento da qualidade nos serviços públicos de saúde.

Qualidade da Assistência à Saúde; Serviços de Saúde; Pessoal de Saúde; Gestão de Qualidade; Planejamento em Saúde

\section{Colaboradores}

L. F. Volpato participou da concepção e planejamento, coleta de dados, análise e interpretação dos dados, redação do artigo para submissão à publicação. M. C. Meneghim contribuiu para a concepção e planejamento do estudo, revisão crítica do conteúdo. A. C. Pereira contribuiu na revisão final do texto. G. M. B. Ambrosano contribuiu na revisão final do texto.

\section{Agradecimentos}

À Coordenação de Aperfeiçoamento de Pessoal de Nível Superior (CAPES), por conceder bolsa de estudo que viabilizou a realização deste estudo, e à Secretaria de Saúde do Município de Piracicaba, pela autorização na realização desta pesquisa.

\section{Referências}

1. World Health Organization. Constitution of the World Health Organization. Basic documents. Geneva: World Health Organization; 1946.

2. Brasil. Lei $n^{\circ}$. 8.080, de 19 de setembro de 1990 . Dispõe sobre as condições para a promoção, proteção e recuperação da saúde, a organização e o funcionamento dos serviços correspondentes e dá outras providências. Diário Oficial da União 1990; 20 set.

3. Roncalli AG. O desenvolvimento das políticas públicas de saúde no Brasil e a construção do Sistema Único de Saúde. In: Pereira AC, organizador. Odontologia em saúde coletiva: planejando ações e promovendo saúde. Porto Alegre: Editora Artmed; 2003. p. 28-49.

4. Fadel MAV, Regis Filho GI. Percepção da qualidade em serviços públicos de saúde: um estudo de caso. Revista de Administração Pública 2009; 43:7-22.
5. Matsuda LM, Évora YDM, Boan FS. O método Desdobramento da Função Qualidade - QFD - no planejamento do serviço de enfermagem. Rev Latinoam Enferm 2000; 8:97-105.

6. Munro ACM. Divergências entre as expectativas do usuário e a percepção da gerência em relação a qualidade do serviço [Dissertação de Mestrado]. Porto Alegre: Universidade Federal do Rio Grande do Sul; 1994.

7. Paiva SMA, Gomes ELR. Assistência hospitalar: avaliação da satisfação dos usuários durante seu período de internação. Rev Latinoam Enferm 2007; 15:973-9.

8. Villela WV, Araújo EC, Ribeiro AS, Cuginotti AP, Hayana ET, Brito FC, et al. Desafios da atenção básica em saúde: a experiência deVila Mariana, São Paulo, Brasil. Cad Saúde Pública 2009; 25:1316-24. 
9. van Stralen CJ, Belisário AS, Stralen TBS, Lima AMD, Massote AW, Oliveira CL. Percepção dos usuários e profissionais de saúde sobre atenção básica: comparação entre unidades com e sem saúde da família na Região Centro-Oeste do Brasil. Cad Saúde Pública 2008; 24 Suppl 1:S148-58.

10. Vasconcelos SM, Bosi MLM, Pontes RJS. Avaliação e monitoramento da atenção básica no Estado do Ceará, Brasil: explorando concepções e experiências no nível central. Cad Saúde Pública 2008; 24:2891-900.

11. Miguel PAC, Weidmann A. Construção da casa da qualidade: exemplo didático para o ensino do desdobramento da função qualidade. Revista de Ensino de Engenharia 1999; 18:41-50.

12. Chaplin E, Bailey M, Crosby R, Gorman D, Holland $\mathrm{X}$, Hippe $\mathrm{C}$, et al. Using quality function deployment to capture the voice of the customer and translate it into the voice of the provider. Jt Comm J Qual Improv 1999; 25:300-15.

13. Porto MBS. Avaliação de processo em hospitais: uma abordagem pelos princípios do QFD [Dissertação de Mestrado]. Florianópolis: Faculdade de Engenharia de Produção, Universidade Federal de Santa Catarina; 1999.

14. Cheng LC. Planejamento da qualidade no TQC. In: Cheng LC, Scapin CA, Oliveira CA, Krafetuski E, Drumond FB, Boan FS, et al., organizadores. QFD: planejamento da qualidade. Belo Horizonte: Líttera Maciel; 1995. p. 3-17.

15. Akao Y. Introdução ao desdobramento da qualidade. Belo Horizonte: Fundação Christiano Ottoni; 1996.

16. Carnevalli JA, Sassi AC, Miguel PAC. Aplicação do QFD no desenvolvimento de produtos: levantamento sobre seu uso e perspectivas para pesquisas futuras. Gestão \& Produção 2004; 11:33-49.

17. Curry A, Herbert D. Continuous improvement in public services: a way forward. Managing Service Quality 1998; 8:339-49.

18. Barley SR. Tecnology as an occasion for structuring: evidence from observations of CT scanners and social order of radiology department. Administrative Science Quarterly 1986; 31:78-108.
19. Gurgel Junior GD, Vieira MMF. Qualidade total e administração hospitalar: explorando disjunções conceituais. Ciênc Saúde Coletiva 2002; 7:325-33.

20. Chan LK, Wu ML. Quality function deployment: A literature review. Eur J Oper Res 2002; 143:463-97.

21. Pochmann M, Amorim R. Atlas da exclusão social no Brasil. 4a Ed. São Paulo: Cortez Editora; 2007.

22. Volpato LF, Meneghim MC, Tengan C, Meneghim ZMAP. Avaliação da qualidade: a possibilidade do uso de uma ferramenta de avaliação no serviço de saúde pública. In: Pereira AC, organizador. Tratado de saúde coletiva em odontologia. Nova Odessa: Napoleão Editora; 2009. p. 111-30.

23. Milan M, Barros JWD, Gava JL. Planning soil tillage using Quality Function Deployment (QFD). Scientia Agricola 2003; 60:217-21.

24. Tengan C. Abordagem teórica e aplicação de um método de qualidade em serviço público odontológico [Tese de Doutorado]. Piracicaba: Faculdade de Odontologia de Piracicaba, Universidade Estadual de Campinas; 2008.

25. Kloetzel K, Bertoni AM, Irazoqui MC, Campos VPG, Santos RN. Controle de qualidade em atenção primária à saúde. I - A satisfação do usuário. Cad Saúde Pública 1998; 14:623-8.

26. Mendes ACG, Araújo Junior JLCA, Furtado BMAS, Duarte PO, Santiago RF, Costa TR. Avaliação da satisfação dos usuários com a qualidade do atendimento nas grandes emergências do Recife, Pernambuco, Brasil. Rev Bras Saúde Matern Infant 2009; 9:157-65.

27. Schwartz TD, Ferreira TB, Maciel ELN, Lima RCD. Estratégia saúde da família: avaliando o acesso ao SUS a partir da percepção dos usuários da unidade de saúde de resistência, na região de São Pedro, no município de Vitória/ES. Ciênc Saúde Coletiva; no prelo.

28. Franco SC, Campos GW. Avaliação da qualidade de atendimento ambulatorial em pediatria em um hospital universitário. Cad Saúde Pública 1998; 14:61-70.

Recebido em 02/Fev/2010

Versão final reapresentada em 10/Jun/2010 Aprovado em 21/Jun/2010 